\title{
Estimation de la ration alimentaire optimale chez les alevins de Parachanna obscura (Perciformes, Channidae)
}

\author{
Diane KPOGUE ${ }^{1}$, Michel SEZONLIN ${ }^{2}$, Herbert HOUEDETE ${ }^{1}$ et Emile FIOGBE ${ }^{1 *}$ \\ ${ }^{I}$ Unité de Recherches sur les Zones Humides, Département de Zoologie et Génétique, Faculté des Sciences et \\ Techniques, Université d'Abomey - Calavi, B.P. 526 Cotonou, Bénin. \\ ${ }^{2}$ Laboratoire de Génétique, Département de Zoologie et Génétique, Faculté des Sciences et Techniques, \\ Université d'Abomey - Calavi, BP. 526 Cotonou, Bénin. \\ *Auteur correspondant; E-mail : edfiogbe@yahoo.fr
}

\section{RESUME}

Parachanna obscura est un poisson carnivore à grande valeur commerciale rencontré au Bénin et dans plusieurs pays africains. Sa domestication serait très utile pour la pisciculture africaine. Or, la domestication d'une espèce piscicole passe par la détermination de ses besoins alimentaires. L'objectif de la présente étude est d'estimer la ration alimentaire optimale chez les alevins de $P$. obscura. Ainsi, après une période d'acclimatation à l'alimentation artificielle, des alevins de $P$. obscura de poids moyen initial 2,45 $\pm 0,09 \mathrm{~g}$, récoltés dans le milieu naturel, ont été soumis à trois rations alimentaires (5,10 et $15 \%$ de la biomasse). L'aliment utilisé a été le Coppens. Chaque ration a été testée en triplicat en circuit ouvert pendant 28 jours. Aucune mortalité n'a été enregistrée. Le poids moyen final et le taux de conversion spécifique les plus élevés ont été obtenus avec la ration 15\% (P > 0,05). Ils ont été respectivement de 5,18 $\pm 0,21 \mathrm{~g}$ et 3,18 $\pm 0,19 \% / \mathrm{J}$. L'efficacité alimentaire a été significativement plus élevée $(\mathrm{P}<0,05)$ avec la ration $5 \%(0,61 \pm 0,07)$. La ration alimentaire optimale peut être estimée à environ $5 \%$ de la biomasse chez les alevins de $P$. obscura (poids moyen initial $\approx 2,45 \mathrm{~g}$ ).

(C) 2011 International Formulae Group. All rights reserved.

Mots clés : Parachanna obscura, alevins, ration optimale, croissance, efficacité alimentaire.

\section{INTRODUCTION}

Le poisson constitue l'une des ressources naturelles les plus précieuses tant dans les pays développés que dans ceux en voie de développement (Kambalé, 1990). De nos jours, on observe une baisse de la productivité halieutique naturelle au niveau de nos plans d'eau à cause des différentes pressions anthropiques (FAO, 2003).
Certaines espèces de poissons dont Parachanna obscura sont alors menacées de disparition (Lalèyè et al., 1997 ; Amoussou, 2003 ; Obasohan, 2008; Babatunde et Olojede, 2010) et leur élevage devient alors impératif. P. obscura est le Channidae africain le plus répandu (Bonou et Teugels, 1985). C'est une espèce à bonne valeur commerciale, grande qualité gastronomique et croissance 
rapide (Dabbadie, 1996) dont la domestication et l'élevage en milieu contrôlé, déjà recommandés par de nombreux auteurs (Micha, 1974 ; Imorou Toko, 2007), n'ont cependant, jamais été essayé. La domestication d'une nouvelle espèce en pisciculture passe tout d'abord par la détermination de ses besoins alimentaires quantitatifs et qualitatifs (Condrey, 1982 ; Imtiaz, 2007). Aussi, la ration alimentaire est l'un des facteurs clés qui influencent la croissance, l'efficacité alimentaire et la survie des poissons d'élevage (Brett et Grove, 1979 ; Reddy et Katre, 1979 ; Reinitz, 1983a, 1983b ; Pickering, 1993; Tacon et Cowey, 1985 ; Talbot, 1985). Par ailleurs, dans toute entreprise aquacole, le volet alimentation constitue près de $70 \%$ des charges (Pillay, 1990). La connaissance des besoins alimentaires quantitatifs d'une espèce permettra sans doute de réduire les coûts de production afin d'assurer une meilleure rentabilité aux entreprises aquacoles. Selon Woods (2005), la connaissance des besoins nutritionnels quantitatifs d'une espèce piscicole permet non seulement de réduire les coûts de production, mais aussi, de minimiser les risques de pollution du milieu d'élevage. De nombreuses études ont été déjà menées sur la biologie et l'écologie de $P$. obscura (Daget, 1950 ; Adebisi, 1987 ; Victor et Okpocha, 1992 ; Teugels et al., 1992 ; Sakiti, 1997). Cependant, ses besoins nutritionnels n'ont jamais été évalués et demeurent inconnus jusque-là. La présente étude dont l'objectif principal est d'estimer la ration alimentaire pouvant induire les performances zootechniques optimales chez les alevins de $P$. obscura élevés en milieu contrôlé au Bénin permettra de combler ce vide.

\section{MATERIEL ET METHODES}

\section{Origine des poissons et conditions expérimentales}

Des alevins de $P$. obscura utilisés au cours de cette expérimentation ont été capturés à l'aide d'une nasse dans un marécage nommé «Dra» situé à Takon dans la commune de Sakété (Sud - Est du Bénin). En période de crue, cette zone entre en communication avec le fleuve Ouémé qui représente le plus grand cours d'eau du pays. Les valeurs moyennes des différents paramètres physico-chimiques de ce milieu ont été de $28,2^{\circ} \mathrm{C} \pm 0,2 ; 6,3 \pm 0,5$ et $2,4 \pm$ $0,3 \mathrm{mg} / \mathrm{L}$, respectivement pour la température, le $\mathrm{pH}$ et le taux d'oxygène dissous.

Après leur capture, les alevins ont été transportés à la station de recherche en Aquaculture de l'Unité de Recherche sur les Zones Humides de la Faculté des Sciences et Techniques de l'Université d'AbomeyCalavi. Le démarrage de l'expérimentation proprement dite a été précédé d'une phase d'acclimatation progressive des alevins aux conditions physico-chimiques de ce nouveau milieu et à l'alimentation artificielle. Au cours de cette période, ils ont été nourris pendant les deux premiers jours avec du zooplancton (asticots vivants). A partir du $3^{\text {ème }}$ jour, le zooplancton a été substitué progressivement par du Coppens de diamètre $0,8 \mathrm{~mm}$. Du $3^{\text {ème }}$ au $4^{\text {ème }}$ jour, $25 \%$ de leur ration a été constituée de Coppens alors qu'au cours des $5^{\text {ème }}$ et $6^{\text {ème }}$ jours, ils ont reçu respectivement 50 et $75 \%$ de Coppens dans leur ration. Dès le $7^{\text {ème }}$ jusqu'au $14^{\text {ème }}$ jour de la phase d'acclimatation à l'aliment artificiel, les alevins ont été nourris exclusivement (100\%) au Coppens.

$\mathrm{Au}$ terme de cette période d'acclimatation, 108 alevins de $P$. obscura de poids et longueur moyens initiaux respectifs de 2,45 g $\pm 0,09 \mathrm{~g}$ et de $6,83 \pm 0,1 \mathrm{~cm}$ ont été répartis dans 9 bassins à raison de 12 alevins par bassin. Les bassins sont de formes rectangulaires disposés en série de dimensions (1,2 X 1,06 X 0,66 m) remplis d'eau au 2/3 de leur volume. Ces bassins ont été entièrement couverts de claies. L'eau des bassins était continuellement renouvelée pendant toute la durée de l'essai avec l'eau courante provenant 
du château d'eau de l'Université d'AbomeyCalavi. La température moyenne de l'eau a été de 28,51 $\pm 0,37{ }^{\circ} \mathrm{C}$. Le $\mathrm{pH}$ et le taux moyen d'oxygène dissous de l'eau ont été respectivement de $5,65 \pm 0,43$ et 5,05 $\pm 0,2$ $\mathrm{mg} / \mathrm{L}$.

Trois (3) rations alimentaires à savoir 5,10 et $15 \%$ de la biomasse ont été testées en triplicat pendant 28 jours. L'aliment utilisé a été le Coppens de diamètre 1,2 $\mathrm{mm}$. Cet aliment contient environ $45 \%$ de protéines, $10 \%$ de lipides et $18,5 \mathrm{Kj} / \mathrm{g}$ d'Energie Brute. L'aliment a été distribué manuellement $a d$ libitum et 5 fois / jour entre 8 et 18 heures.

Des pêches de contrôle ont été faites chaque semaine afin d'estimer l'évolution du poids moyen et d'ajuster la ration à la biomasse. La biomasse totale de tous les alevins par bassin a été alors mesurée à chaque pêche de contrôle. Au début et à la fin de l'expérience, le nombre total d'alevins et la biomasse par bassin ont été mesurés. Les poids et longueurs individuels de tous les poissons ont été également mesurés par bassin.

\section{Evaluation des performances de chaque traitement}

Les paramètres suivants ont été calculés pour chaque traitement :

$\mathrm{TCS}=100(\mathrm{LnPf}-\mathrm{LnPi}) / \Delta \mathrm{t} ; \mathrm{EA}=(\mathrm{Bf}-$ $\mathrm{Bi}) / \mathrm{RD} ; \mathrm{TS}=100 \times \mathrm{Nf} / \mathrm{Ni} ; \mathrm{K}(\%)=\left(\mathrm{Pf} / \mathrm{L}^{3}\right) \mathrm{x}$ $100 ; \mathrm{G}=\mathrm{Pf}-\mathrm{Pi} ; \mathrm{PP}=\mathrm{PRC} / \mathrm{G}$.

TCS = Taux de croissance spécifique $(\% / \mathrm{J}), \mathrm{LnPf}=$ logarithme népérien du poids final, $\mathrm{LnPi}=$ logarithme népérien du poids initial, $\Delta \mathrm{t}=$ durée de l'expérience, $\mathrm{EA}=$ Efficacité Alimentaire, $\mathrm{Bf}=$ Biomasse finale, $\mathrm{Bi}=$ Biomasse initiale, $\mathrm{RD}=$ Ration Distribuée, $\mathrm{TS}=$ Taux de Survie, Nf = Nombre final, $\mathrm{Ni}=$ Nombre initial, $\mathrm{L}$ : longueur finale à la fourche, $\mathrm{G}=$ Gain de poids individuel, $\mathrm{Pf}=$ Poids final, $\mathrm{Pi}=$ Poids initial, PP = Prix du Prégrossissement, PRC = Prix de Revient du Coppens par alevin.

\section{Analyse statistique des données}

L'analyse statistique des résultats obtenus a été effectuée à l'aide du logiciel Statistica 5.5 par la méthode d'analyse de variance à un critère de classification (ANOVA 1). Le test de Hartley (1959) nous a permis de tester l'homogénéité des variances (Dagnelie, 1975). Le test de LSD (Least Significant Difference) (Saville, 1990), nous a permis de déterminer les différences entre les résultats obtenus pour chaque paramètre calculé en fonction des rations testées. Un seuil de probabilité de 5\% a été retenu.

\section{RESULTATS}

Le Tableau 1 montre l'effet des différents traitements sur les principaux paramètres zootechniques.

La Figure 1 montre l'évolution des poids des alevins en fonction des traitements. Le poids moyen final le plus élevé a été obtenu avec le traitement $\mathrm{T} 3$ où les poissons ont été nourris à $15 \%$ de leur biomasse/J. Aucune différence significative $(p>0.05)$ n'a été cependant mise en évidence entre les différents poids finaux.

Les taux de croissance spécifique (TCS) augmentent quand la ration alimentaire croît (Tableau 1). Cependant, aucune différence significative n'a été montrée entre les taux de croissance spécifique obtenus pour chacun des trois différents traitements ( $\mathrm{P}>0,05)$.

L'efficacité alimentaire obtenue avec le traitement $\mathrm{T} 1$ (5\% de la biomasse) a été significativement supérieure à celle des deux autres traitements $(\mathrm{p}<0,05)$ (Figure 2).

Le Tableau 2 montre que le coût de prégrossissement des alevins nourris à $5 \%$ de leur biomasse est significativement plus faible et économiquement plus rentable que celui des deux autres traitements $(\mathrm{p}<0,05)$. 
Tableau 1 : Influence des différents traitements (rations alimentaires) sur les principaux paramètres zootechniques au bout des 28 jours de traitement.

\begin{tabular}{lllll}
\hline Paramètres & & T1 (5\%) & T2 (10\%) & T3 (15\%) \\
\hline Nombre & initial & 12 & 12 & 12 \\
& final & 12 & 12 & 12 \\
\hline Poids moyen (g) & initial & $2,5 \pm 0,22 \mathbf{a}$ & $2,42 \pm 0,14 \mathbf{a}$ & $2,42 \pm 0,08 \mathbf{a}$ \\
& final & $4,40 \pm 0,02 \mathbf{a}$ & $4,42 \pm 0,08 \mathbf{a}$ & $5,18 \pm 0,21 \mathbf{a}$ \\
\hline Taux de croissance spécifique (TCS) (\%/J) & $2,37 \pm 0,35 \mathbf{a}$ & $2,52 \pm 0,26 \mathbf{a}$ & $3,18 \pm 0,19 \mathbf{a}$ \\
Efficacité alimentaire (EA) & $0,61 \pm 0,07 \mathbf{a}$ & $0,29 \pm 0,04 \mathbf{b}$ & $0,28 \pm 0,01 \mathbf{b}$ \\
Taux de survie (\%) & $100 \pm 0,00 \mathbf{a}$ & $100 \pm 0,00 \mathbf{a}$ & $100 \pm 0,00 \mathbf{a}$ \\
Coefficient de condition (\%) & $0,86 \pm 0,04 \mathbf{a}$ & $0,80 \pm 0,04 \mathbf{a}$ & $0,73 \pm 0,02 \mathbf{a}$ \\
\hline
\end{tabular}

Les valeurs se trouvant sur la même ligne et partageant la même lettre ne sont pas significativement différentes ( $p>0,05)$.

Tableau 2 : Estimation du coût de prégrossissement par alevin au bout de 28 jours.

\begin{tabular}{|c|c|c|c|}
\hline Paramètres $\quad$ Traitement & $\mathbf{T 1}(5 \%)$ & $\mathrm{T} 2(10 \%)$ & T3 (15\%) \\
\hline Poids moyen individuel (g) & $2,5 \pm 0,22$ & $2,42 \pm 0,14$ & $2,42 \pm 0,08$ \\
\hline final & $4,40 \pm 0,02 \mathrm{a}$ & $4,42 \pm 0,08 \mathrm{a}$ & $5,18 \pm 0,21 \mathrm{a}$ \\
\hline Gain de poids individuel $(\mathrm{g})$ & $1,90 \pm 0,20 \mathrm{a}$ & $2,00 \pm 0,06 \mathrm{a}$ & $2,76 \pm 0,13 a$ \\
\hline Quantité d'aliment distribuée/alevin (g) & $3,12 \pm 0,34$ & $6,83 \pm 0,50$ & $10,01 \pm 0,49$ \\
\hline Prix du "Coppens" (F.CFA/g) & 3,5 & 3,5 & 3,5 \\
\hline Prix de revient du "Coppens" / alevin (F.CFA)* & $10,92 \pm 1,19 \mathrm{a}$ & $23,90 \pm 1,75 b$ & $35,03 \pm 1,71 b$ \\
\hline Prix de prégrossissement / g d'alevin (F.CFA/g) & $5,75 \mathrm{a}$ & $11,95 \mathrm{~b}$ & $12,69 \mathrm{~b}$ \\
\hline
\end{tabular}

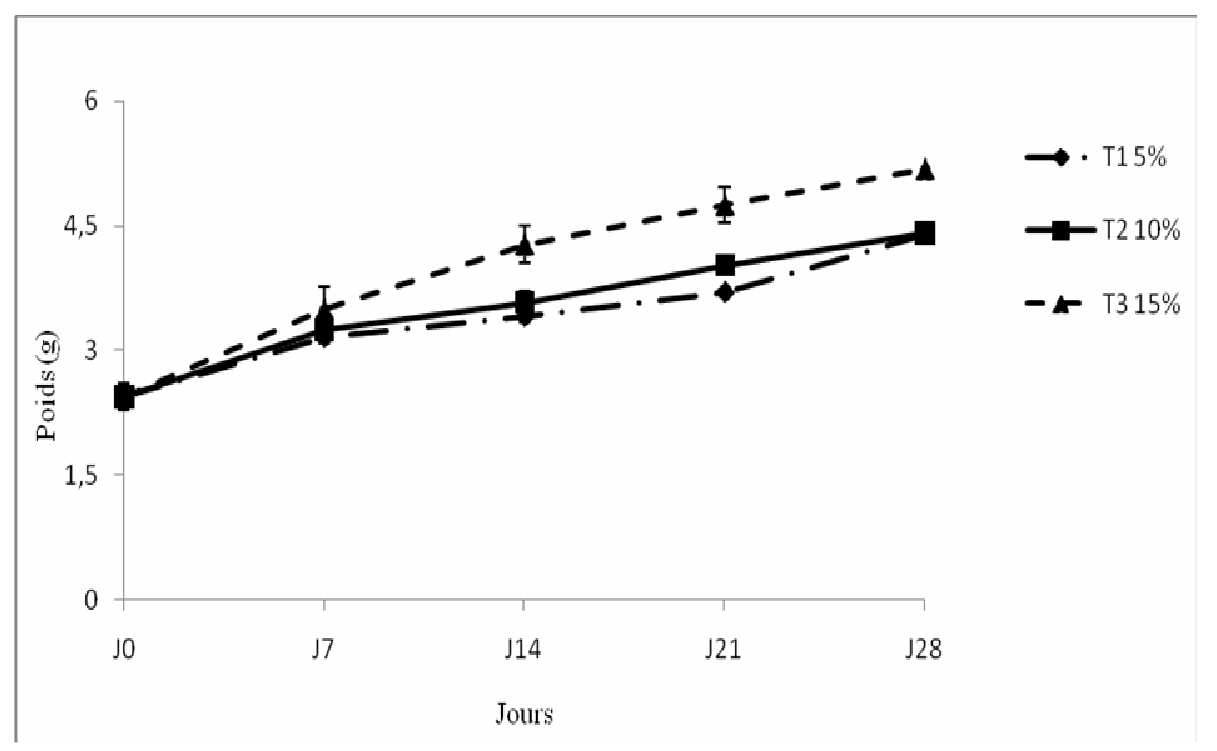

Figure 1 : Evolution des poids individuels moyens en fonction des taux de rationnement. 


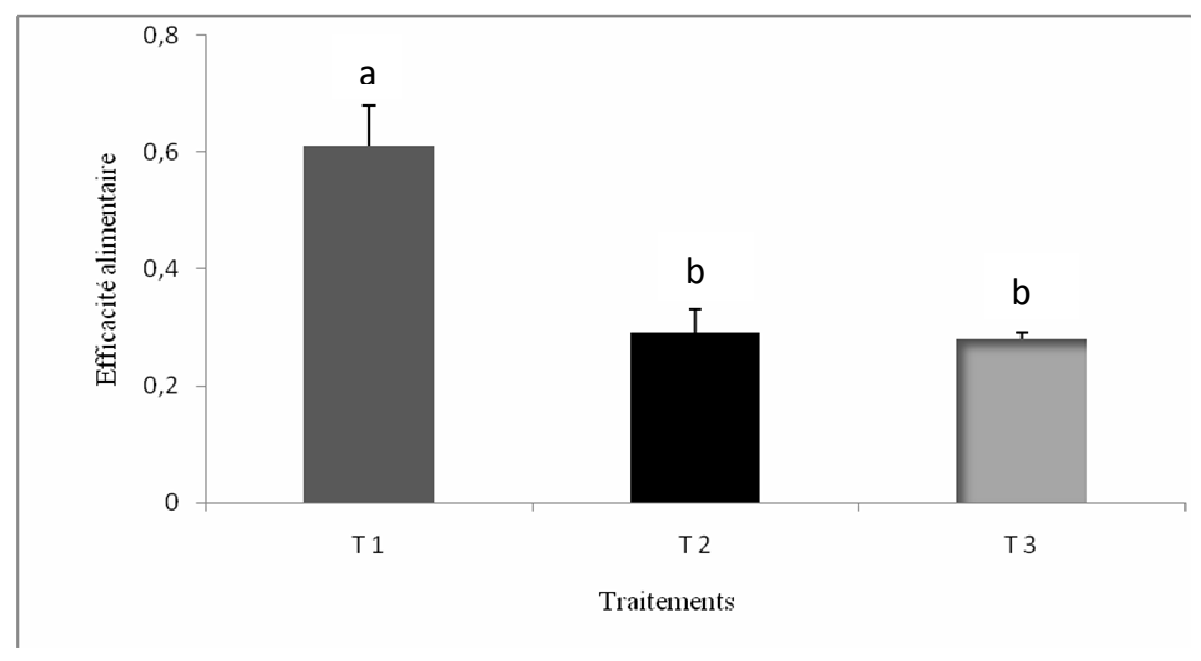

Figure 2 : Variation de l'efficacité alimentaire en fonction des taux de rationnement.

\section{DISCUSSION}

Aucune mortalité n'a été enregistrée au cours de cet essai. Les rations alimentaires testées n'ont pas alors affecté la survie des poissons.

La croissance des poissons s'est améliorée au fur et à mesure que la ration alimentaire est augmentée. Les taux de croissance spécifiques (TCS) moyens obtenus pour tous les traitements $(2,37 \pm 0,35 ; 2,52 \pm$ $0,26$ et $3,18 \pm 0,19 \% / J)$ sont proches des 3 $\% / \mathrm{J}$ recommandés en aquaculture pour les alevins de poissons carnivores (Barnabé, 1991). Ils sont aussi proches des 2,63 et 3,10 $\% / \mathrm{J}$ obtenus respectivement par Mélard et al. (1995) et Fiogbé (1996) sur des alevins de $3 \mathrm{~g}$ de Perca fluviatilis, et Nyinawamwiza (2007) sur des alevins de $4 \mathrm{~g}$ de Clarias gariepinus.

L'efficacité alimentaire a varié de 0,61 $\pm 0,07(\mathrm{~T} 15 \%)$ à $0,28 \pm 0,01$ (T3 15\%). Ces valeurs sont relativement faibles. Autrement dit, l'aliment n'a pas été bien converti par les poissons. Cela pourrait s'expliquer par la faible densité de mise en charge ou par le besoin optimal en protéines chez les alevins de $P$. obscura qui serait différent des $45 \%$ contenus dans Coppens. En effet, si la densité est plus petite que la densité optimale, on assiste au gaspillage et à une mauvaise utilisation des aliments qui se traduit par des efficacités alimentaires faibles (Jobling,
1985). Par ailleurs, si le besoin protéique optimal des alevins de P.obscura est différent de $45 \%$, alors il y aura manque considérable de l'énergie destinée à la croissance (Houlihan, 1991) et les efficacités alimentaires seront relativement faibles.

La ration alimentaire optimale étant celle qui associe ration alimentaire minimale et l'efficacité alimentaire maximale pour une meilleure rentabilité économique, la ration alimentaire optimale chez les nalevins de $P$. obscura de poids moyen individuel 2,5 g serait d'environ 5\% de la biomasse.

Ces résultats sont en adéquation avec ceux de Lequenne (1984) et Fiogbé (1996, 2003) selon lesquels la ration alimentaire optimale varie de 1 à $5 \%$ chez alevins de poissons carnivores de poids initial supérieur à $1 \mathrm{~g}$.

\section{Conclusion}

Au terme de cette étude, les taux de survie et de croissance spécifique observés pour tous les traitements témoignent de la rusticité et de la bonne adaptation des alevins de $P$. obscura aux conditions d'élevage en milieu contrôlé. L'analyse économique des performances de chaque traitement montre que la ration alimentaire optimale chez les alevins de P.obscura de poids moyen initial 
de 2,5 g est d'environ $5 \%$ de la biomasse totale.

Des recherches complémentaires sur l'estimation des rations alimentaires des alevins de cette espèce et de ce même poids permettront de préciser sa ration alimentaire optimale et ses rations d'entretien et maximale pour une meilleure utilisation de l'aliment.

\section{REMERCIEMENTS}

Nous remercions le Ministère de l'Enseignement Supérieur et de la Recherche Scientifique de la République du Bénin qui a octroyé une bourse doctorale à Diane KPOGUE et Mr Marius qui nous a aidé lors de la capture des alevins.

\section{REFERENCES}

Adebisi AA. 1987. The relationship between the fecundities, gonado-somatic indices and egg sizes of some fishes of Ogun River, Nigeria. Hydrobiologia, 3: 151156.

Amoussou E. 2003. Dynamique hydrosédimentaire et mutations des écosystèmes du lac Ahémé. Mémoire de Maîtrise, Université d'Abomey-Calavi. $103 \mathrm{p}$.

Babatunde EE, Olojede ET. 2010. Comparative studies on the catch selectivity of galvanized wire gauze trap from fresh and brackish water tropical creeks in south-western Nigeria. Journal of life \& Physical Sciences, 3(2): 91-101.

Barnabé G. 1991. Bases Biologiques et Ecologiques de l'Aquaculture. Coll. TEC \& DOC. Lavoisier ; 489 p.

Bonou CA, Teugels GG. 1985. Révision systématique du genre Parachanna (Pisces: Channidae). Revue d'Hydrobiologie Tropicale, 18: 267-280.

Brett JR, Groves TDD. 1979. Fish physiology. Physiological Energetics, 3: 280-282.

Condrey RE. 1982. Ingestion - limited growth of aquatic animals: the case for Blackman kinetics. Canadian Journal of Fisheries Aquatic Science, 39: 1585-1595.
Dabbadie ML. 1996: Etude de la viabilité d'une pisciculture rurale à faible niveau d'intrant dans le Centre-Ouest de la Côte d'Ivoire : Approche du réseau trophique. Thèse de doctorat de l'Université de Paris $6,214 p$.

Daget J. 1950. Poissons d'eau douce de la région côtière du Togo et du Dahomey. Notes Africaines, 46: 57-59.

Dagnelie P. 1975. Théorie et Méthodes Statistiques (Vol. II). Presses Agronomiques de Gembloux: Belgique ; $463 \mathrm{p}$.

FAO. 2001. Promotion de l'aquaculture commerciale durable en Afrique subsaharienne. Expériences de certains pays en développement, 293p.

Fiogbé ED. 1996. Contribution à l'étude des besoins nutritionnels chez les larves et juvéniles de la perche fluviatile (Perca fluviatilis L.). Thèse de Doctorat, FUNDP, Namur, 288 p.

Fiogbé ED, Kestemont P. 2003. Optimum daily ration for Eurasian perch Perca fluviatilis L. reared at its optimum growing temperature. Aquaculture, 216: 243-252.

Hartley HO. 1959. Smallest composite designs for quadratic response surface. Biometrics, 15: 611- 624.

Houlihan DF. 1991. Protein Turnover in Ectotherms and its Relationships to Energetic. In Advances in Comparative and Environmental Physiology (vol. 7), Gilles R (ed). Springer Verlag: Berlin; 1-43.

Imorou Toko I. 2007. Amélioration de la production halieutique des trous traditionnels à poissons (whedos) du delta de l'Ouémé (Sud-Bénin) par la promotion de l'élevage des poissons-chats Clarias gariepinus et Heterobranchus longifilis. Thèse de doctorat, FUNDP, Namur, $214 \mathrm{p}$.

Imtiaz A. 2007. Effect of ration size on growth, body composition, and energy and protein maintenance requirement of fingerling Indian major carp, Labeo 
rohita (Hamilton). Fish Physiology and Biochemistry, 33: 203- 212.

Jobling, M. 1985. Physiological and social constraints on growth of fish with special reference to Arctic charr Salvelinus alpinus L. Aquaculture, 44: 83-90.

Kambale K. 1990. Contribution à l'étude du rythme d'activité des poissons de la rivière Tshopo et du fleuve Zaïre à Kisangani. Mémoire en Sciences, 55 p.

Lalèyè P, Chikou A, Wuemènou. T. 1997. Poissons d'eaux douces et saumâtres du Bénin : inventaire, distribution, statut et conservation. Inventaire des poissons menacés de disparition, statut et conservation. Inventaire des poissons menacés de disparition du Bénin. Rapport d'étude, Coopération Béninonéerlandaise/Ambassade royale des PaysBas, Cotonou, Bénin, 80 p.

Mélard C, Abi-Ayad A, Grignard JC, Baras, E, Paelinck P, Kaiser L, Philippart JC, Kestemont P, Fiogbé ED, Pirmez L, Micha JC. 1995. Diversification de la pisciculture Wallonne. Mise au point de l'élevage intensif de nouvelles espèces de consommation et de repeuplement cyprinidés et percidés. Rapport de recherche à la Région Wallonne, Université de Liège, FUNDP. Namur. $58 \mathrm{p}$.

Micha JC. 1974. Fish populations study of Ubangui River: Trying local wild species for fish culture. Aquaculture, 4: 85-87.

Nyinawamwiza L. 2007. Valorisation des sous-produits agro-industriels dans l'élevage du poisson-chat africain Clarias gariepinus au Rwanda: Effet sur les performances de croissance et de reproduction. Thèse de doctorat, FUNDP. Namur, $188 \mathrm{p}$.

Obasohan EE. 2008. Fisheries biodiversity: The role of a traditional taboo/ ritual prohibition in the management and conservation of the fish resources of
Ibiekuma Stream in Ekpoma, Edo State, Nigeria. Bioscience Research Communications, 20: 257- 264.

Pickering AD. 1993. Growth and stress in fish production. Aquaculture, 111: 51- 63.

Pillay TVR. 1990. Aquaculture: Principles and Practices. Fishing News Books: Oxford; $575 \mathrm{p}$.

Reddy SR, Katre S. 1979. Growth rate and conversion efficiency of the air-breathing catfish, Heteropneustes fossilis, in relation to ration size. Aquaculture, 18: $35-40$.

Reinitz G. 1983a. Relative effect of age, diet and feeding rate on the body composition of young rainbow trout (Salmo gairdneri). Aquaculture, 35: 19 -27.

Reinitz G. 1983b. Influence of diet and feeding rate on the performance and production costs of rainbow trout. Trans American Fisherie Society, 112: 830-833.

Sakiti GN. 1997. Myxosporidies et Microsporidies de poissons du Sud Bénin : Faunistiques, Ultrastrutures, Biologie. Thèse de Doctorat d'Etat ès sciences de Biologie animale, Université Nationale du Bénin, Bénin, 296p.

Saville DJ. 1990. Multiple comparison procedures: the practical solution. American Statistic., 44(2): 174-180.

Teugels GG, Reid GM, King RP. 1992. Fishes of the Cross River basin (Cameroon Nigeria) taxonomy, zoogeography, ecology and conservation: Tervuren Belgique, Musée Royale de l'Afrique Centrale. Annales Sciences Zoologiques, 266: 132.

Victor R, Akpocha BO. 1992. The biology of Snakehead, Channa obscura (Gunther), in a Nigerian pond under monoculture. Aquaculture, 101: 17- 24.

Woods CMC. 2005. Growth of cultured seahorses (Hippocampus obdominalis) in relation to feed ration. Aquaculture International, 13: 305-314. 